\title{
Determination of Partial Discharge Inception Voltage and Location of Partial Discharges by Means of Paschen's Theory and FEM
}

\author{
Guillaume Parent ${ }^{1}$, Mathieu Rossi ${ }^{1}$, Stéphane Duchesne ${ }^{1}$ and Patrick Dular ${ }^{2}$ \\ ${ }^{1}$ Univ. Artois, EA 4025, Laboratoire Systèmes Électrotechniques et Environnement (LSEE), F-62400 Béthune, France \\ ${ }^{2}$ Department of Electrical Engineering and Computer Science, Montefiore Institute, University of Liège, B-4000 Liège, Belgium \\ guillaume.parent@univ-artois.fr
}

Determining the Partial Discharge Inception Voltage (PDIV) is a key point when designing electrical coils or busbars. In this paper, a method making it possible to calculate the PDIV as well as to locate partial discharges in windings of any shape is presented. It is based on a 2D finite element computation using both dual - i.e. scalar and vector potential - electrostatic formulations and a comparison of the obtained operating points with the Paschen's curve.

Index Terms-Finite element method, field lines, partial discharge inception voltage.

\section{INTRODUCTION}

D EPENDING on the applications they are used for, electrical machines are subject to different stress factors, such as thermal, mechanical and electrical stresses. They all contribute to the machine possible failures [1]. Among them, $30 \%$ are related to electrical faults, the majority of which coming from a windings insulation breakdown [1]. It is highlighted in the literature [2] that Partial Discharge (PD) appearance is responsible for the insulation system early aging. This is the reason why determining a priori the Partial Discharge Inception Voltage (PDIV) is a key point when designing electrical windings. So far, only experimental tests realized on already built devices allow to determine this quantity, which conflict with a design step.

It is reported in the literature that the Paschen's law $[3,4]$ is an efficient criteria for the PDIV determination of electrical machines windings $[2,5,6]$. To be utilized, this criteria requires two quantities. The first one is the distance of the path covered by free electrons during the avalanche breakdown process. In Paschen's theory, this path can be assimilated to an electric field line. The second quantity is the electric potential difference between the two ends of that field line. Although determining those two quantities is relatively easy in the case of two plane electrodes, it is still impossible in the case of more complex shapes [6].

The aim of this paper is to provide a numerical method allowing to treat a priori any 2D complex geometry - cables, motor/transformer windings or busbars - whether for the determination of the PDIV or the location of PDs. It is shown that using both dual - scalar and vector potential electrostatic formulations allows to account for the correct pair of aforementioned quantities, which, to the author's knowledge, is an approach that has never been seen in the literature. Three application examples are treated in order to evaluate both the validity and the robustness of the method.

All FEM computations are performed using the code GetDP [7]. Its Open Source approach allowed us to implement the electric vector potential formulation which, to the authors' knowledge, is never implemented in any commercial software, as well as the post-operations allowing to compute the PDIV.

\section{PASChEn's LaW For the Determination OF DISCHARGE PRECURSORS}

In [3], Friedrich Paschen described the theory which allows to determine the $V_{\mathrm{b}}$ voltage corresponding to the initiation of phenomena leading to the electrical breakdown of a motionless gas located between two plane electrodes. This breakdown voltage is governed by [4]:

$$
V_{\mathrm{b}}=\frac{B p d}{\ln (A p d)-\ln \left[\ln \left(1+\frac{1}{\gamma_{\mathrm{se}}}\right)\right]}
$$

where $p$ is the pressure (in Torr), $d$ is the distance (in $\mathrm{cm}$ ) between either the electrodes or the insulation layers if any, $\gamma_{\mathrm{se}}$ is the Townsend secondary electron emission coefficient and $A$ and $B$ are coefficients which depend on the considered gas and temperature $[3,6]$. Then, it is possible to plot the so-called Paschen's curve $V_{\mathrm{b}}=f(d)$ (Fig. 1).

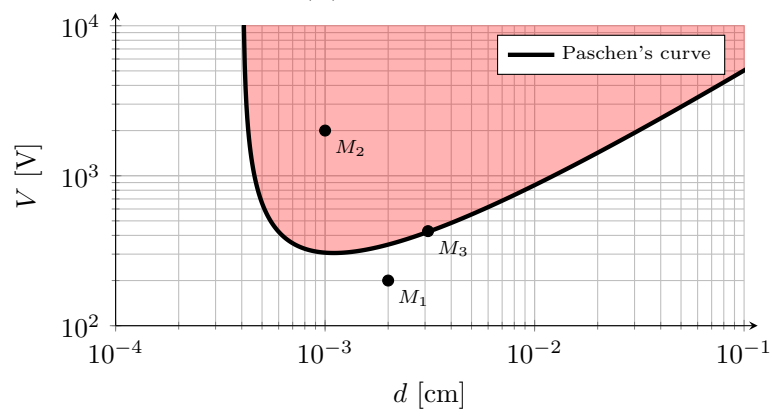

Fig. 1. Paschen's curve for air at ambient pressure and temperature

This curve can be used as a criteria for the determination of the PDIV $[2,5,6]$. Indeed, let's consider a point, noted $M$, of coordinates $\left(d_{\mathrm{es}}, V_{\mathrm{es}}\right)$ where $d_{\mathrm{es}}$ is the distance between the two insulation layers and $V_{\mathrm{es}}=\left|V_{\mathrm{e}}-V_{\mathrm{s}}\right|$ (Fig. 2a). Then, there are three possibilities:

1) $M$ is located below the curve $\left(M_{1}\right.$ in Fig. 1$)$, which means that no PD appears. 
2) $M$ is located above the curve $\left(M_{2}\right.$ in the red zone in Fig. 1), which means that PDs will appear.

3) $M$ is located on the curve ( $M_{3}$ in Fig. 1), which means that the PDIV is equal to the supply voltage applied to the electrodes.

As previously stated, this theory has been established in [3] from two plane electrodes (Fig. 2a), which implies that the electric field between them is homogeneous. In consequence, in such a configuration, both $V_{\mathrm{es}}$ and $d_{\mathrm{es}}$ are easy to determine. This theory can still be applied to other electrodes shapes $[2,5,6]$, such as circular ones (Fig. 2b). Indeed, PDs appear in areas presenting very high electric field levels inside very small gaps between the electrodes, like between two adjacent turns of a coil for example. For this reason, even if the field is non-homogeneous in this case, the dimensions of considered geometries are such that the electric field along a field line can be considered as uniform, on one hand, and those field lines bending radii are very small, on the other hand, which means that all the assumptions made for rectangular shaped electrodes cases are still valid for circular shaped electrodes cases. Nevertheless, in the latter case, both $V_{\mathrm{es}}$ and $d_{\mathrm{es}}$, which is the length of a curved field line (Fig. 2b), are impossible to determine experimentally.

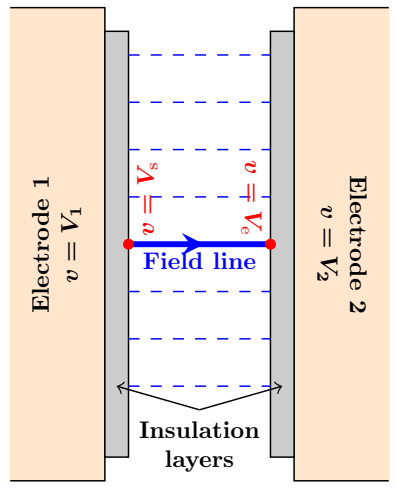

(a) Rectangular shaped electrodes

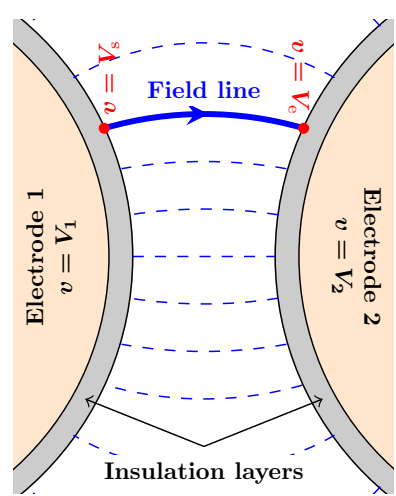

(b) Circular shaped electrodes
Fig. 2. Field lines between two insulated electrodes

\section{Using Both Electrostatic DUAL Formulations FOR THE DETERMINATION OF $V_{\mathrm{B}}$ AND $d$}

An electrostatic problem in a bounded domain $\Omega \in \mathbb{R}^{3}$, of boundary $\Gamma=\Gamma_{\mathrm{e}} \cup \Gamma_{\mathrm{d}}$, is described by the electric field $e$ and the electric flux density $\boldsymbol{d}$, governed by the curl and div equations respectively, and related to each other by a constitutive law as follows:

$$
\begin{gathered}
\operatorname{curl} \boldsymbol{e}=0 \\
\operatorname{div} \boldsymbol{d}=\rho \\
\boldsymbol{d}=\epsilon \boldsymbol{e} \\
\boldsymbol{n} \times\left.\boldsymbol{e}\right|_{\Gamma_{\mathrm{e}}}=0 \\
\left.\boldsymbol{n} \cdot \boldsymbol{d}\right|_{\Gamma_{\mathrm{d}}}=0
\end{gathered}
$$

where $\boldsymbol{n}$ is the unit normal vector exterior to $\Omega, \rho$ is the charge density, $\epsilon$ is the electric permittivity. $\Omega$ is composed of conducting and nonconducting parts noted $\Omega_{\mathrm{c}}$ and $\Omega_{\mathrm{c}}^{\mathrm{C}}$ respectively, with $\Omega=\Omega_{\mathrm{c}} \cup \Omega_{\mathrm{c}}^{\mathrm{C}}$. Source parts and dielectric parts belong to $\Omega_{\mathrm{c}}$ and $\Omega_{\mathrm{c}}^{\mathrm{C}}$ respectively.
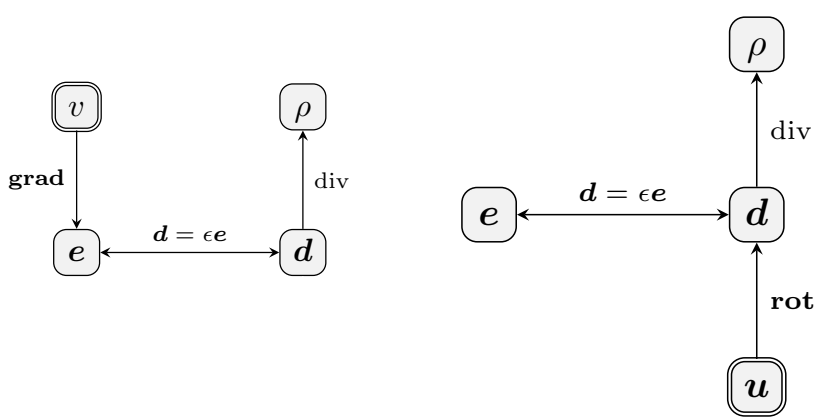

(a) Scalar potential formulation

(b) Vector potential formulation

Fig. 3. Tonti diagrams relative to electrostatic fields

System (2) can be solved by strongly verifying either (2a) or (2b) and then weakly solving the other one. That way, depending on which relation is strongly verified, the two dual formulations can be established. The scalar potential $(v)$ formulation, which allows to strongly verify (2a), is obtained by working with the electric field $e$.

$$
\operatorname{div}(\epsilon \operatorname{grad} v)=\rho
$$

The vector potential formulations is obtained by working with the electric flux density $\boldsymbol{d}$. In this case, the approach is a little bit different since $\boldsymbol{d}$ is not divergence-free in $\Omega_{\mathrm{c}}$. Then, in $\Omega_{\mathrm{c}}^{\mathrm{C}}$, a source field $\boldsymbol{d}_{\mathrm{s}}$ ensuring $\operatorname{div} \boldsymbol{d}_{\mathrm{s}}=\rho$ has to be introduced [8]. The associated reaction field can thus be expressed as the curl of an electric vector potential $\boldsymbol{u}$ and then the electric flux density becomes $\boldsymbol{d}=\boldsymbol{d}_{\mathrm{s}}+$ curl $\boldsymbol{u}$. Combining this relation with (2c) and reporting the result in (2a):

$$
\operatorname{curl}\left(\epsilon^{-1} \boldsymbol{d}_{\mathrm{s}}\right)+\operatorname{curl}\left(\epsilon^{-1} \operatorname{curl} \boldsymbol{u}\right)=\rho
$$

Note that the charge density being a surface one, with zero field in $\Omega_{\mathrm{c}}$, (3) and (4) are to be solved in $\Omega_{\mathrm{c}}^{\mathrm{C}}$, where $\rho=0$.

Since we have two formulations, the question is: which one to use for the determination of $V_{\mathrm{es}}$ and $d_{\mathrm{es}}$ ? Actually, the answer to this question can be found in Tonti diagrams (Fig. 3). Fig. 3a shows that determining $V_{\mathrm{es}}$ is straightforward from $v$ using the scalar potential formulation. Nevertheless, it does not allow for field lines distribution determination without performing an additional post-treatment from the electric field distribution. On the other hand, Fig. 3b shows that the vector potential formulation allows to access to the field lines distribution. Indeed, in $2 \mathrm{D}, \boldsymbol{u}=u \boldsymbol{z}$ where $\boldsymbol{z}$ is the unit vector perpendicular to the studied plane. Then, the electric field can be written as $\boldsymbol{e}=\epsilon^{-1}$ curl $\boldsymbol{u}=\epsilon^{-1}(\operatorname{grad} \mathrm{u}) \times \boldsymbol{z}$ which implies that the field lines are the iso- $u$ lines. Moreover, the vector potential formulation also allows to compute $V_{\mathrm{es}}$ from the electric field by means of its line integral along the considered field line $\int_{\mathrm{s}}^{\mathrm{e}} \boldsymbol{e} \cdot d \boldsymbol{l}$. Nevertheless, as previously stated, (4) does not allow to strongly verify (2a), which means that the line integral of $e$ can suffer from numerical errors.

For all these reasons, as well as due to their complementarity [9], the approach of using the dual formulations is to be prefered. Then, the determination of the PDIV consists in following these steps:

- For the supply voltage applied to the electrodes $\left|V_{2}-V_{1}\right|=1 \mathrm{~V}$, perform an electrostatic finite element 
analysis using (4) in order to compute the $d_{\mathrm{es}}$ length of each of a set of electric field lines in the air parts of $\Omega_{c}^{C}$.

- For the same supply voltage and on the same mesh, perform an electrostatic finite element analysis using (3) in order to compute $V_{\mathrm{es}}$.

- Plot $V_{\mathrm{es}}=g\left(d_{\mathrm{es}}\right)$ and the Paschen's curve $V_{\mathrm{b}}=f\left(d_{\mathrm{es}}\right)$ in the same coordinate system. Due to the low supply voltage, the curve $g$ is way below $f$ at this step.

- Taking advantage of the fact that the problem is linear, compute the scaling factor to apply to the supply voltage that leads to one of the computed $V_{\mathrm{es}}$ to match $V_{\mathrm{b}}$. In other words, compute the scaling factor leading to the two curves $f$ and $g$ to touch each other.

Following this procedure, and since the original supply voltage was intentionally chosen equal to $1 \mathrm{~V}$, the PDIV is equal to the computed scaling factor.

\section{ApPliCATION EXAMPLES}

\section{A. Pair of twisted wires}

The first application example is a pair of identical twisted wires with a diameter of $1.25 \mathrm{~mm}$. The thickness and the relative permittivity of the insulation layers are $60 \mu \mathrm{m}$ and 3.4 respectively (Fig. 4). This testing sample is used for standardized PD measurements (IEC60270 and IEC60851-5). The measured PDIV, obtained according to the procedure described in IEC60270, is equal to $920 \mathrm{~V}$. Fig. 5 presents the curve $V_{\mathrm{es}}=g\left(d_{\mathrm{es}}\right)$ obtained when $V_{\mathrm{es}}$ is computed using the procedure described in the previous Section, i.e. using dual formulations. As a comparison, this figure also presents the curve obtained when only the vector potential formulation is used, which means $V_{\mathrm{es}}$ is computed using the line integral of the electric field, i.e. $V_{\mathrm{es}}=\int_{\mathrm{s}}^{\mathrm{e}} \boldsymbol{e} \cdot d \boldsymbol{l}$. Figure 5 shows that both methods lead to close values of $V_{\mathrm{es}}$ matching the Paschen's curve. This value is $427 \mathrm{~V}$ for the dual formulations procedure and is $447 \mathrm{~V}$ for the line integral from the vector

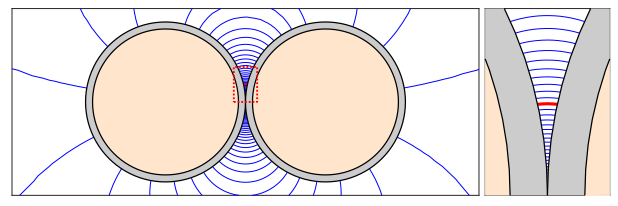

Fig. 4. Field lines distribution in the air surrounding a pair of twisted wires. (left) zoom on the conductors (right) zoom on the critical field line.

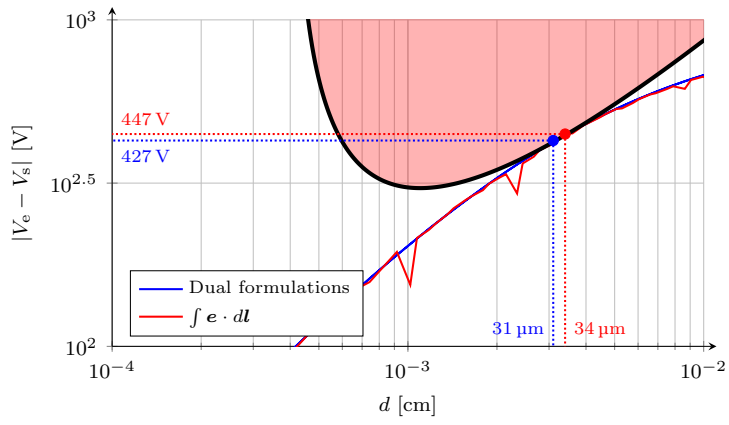

Fig. 5. PDIV determination for a standardized pair of twisted wires potential computation method, which correspond to PDIVs equal to $937 \mathrm{~V}$ and $944 \mathrm{~V}$ respectively. Both values are in very good agreement with the measured value since the error is less than $3 \%$. Nevertheless, the line integral method leads to some numerical errors on the curve presented in Fig. 5 which are due to the fact that the vector potential formulation (4) does not allow to strongly verify (2a). As an illustration, the field lines along which PDs are expected to appear is presented in Fig. 4.

\section{B. Wire wound around on a grounded hollow tube}

The second application example is also usually used for standardized PD measurements. It consists of a wire with a diameter of $1.25 \mathrm{~mm}$ wound around a hollow pipe which is grounded (Fig. 6). The thickness and the relative permittivity of the insulation layers are $60 \mu \mathrm{m}$ and 3.4 respectively. It is an axisymmetric problem that can be solved in 2D. The measured PDIV is equal to $738 \mathrm{~V}$ (Fig. 7).

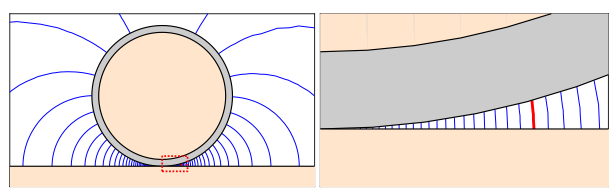

Fig. 6. Field lines distribution in the air surrounding the wire wound around a grounded hollow tube. (left) zoom on the conductor (right) zoom on the critical field line.

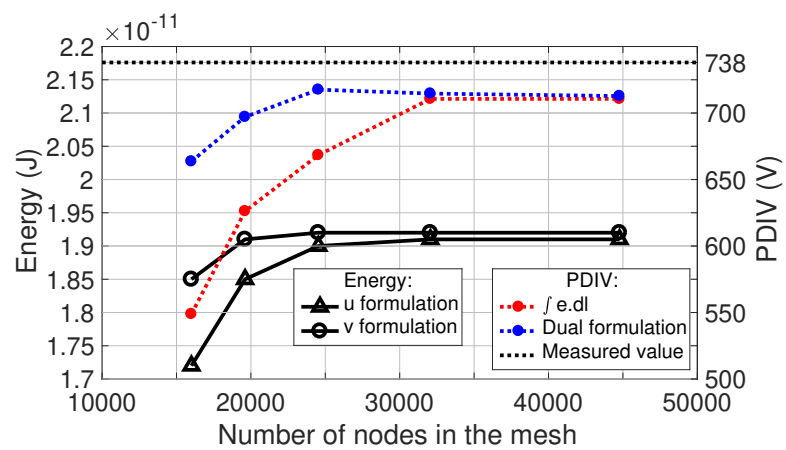

Fig. 7. Energy and PDIV as functions of the number of nodes in the mesh

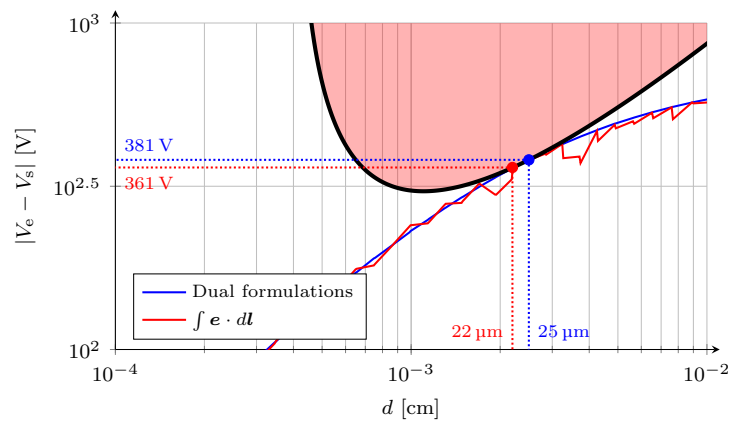

Fig. 8. PDIV determination for a wire wound around a grounded hollow tube

For this study, several meshes, from relatively coarse to very thin ones, were used in order to evaluate the robustness of both the dual formulations and the line integral methods. Fig. 7 presents both the energy and the PDIV as functions of the number of nodes in the used mesh. The energy curve shows 
that using a mesh of more than 253000 nodes lead to a gap between the two formulations is less than $1 \%$, which means that both computation can be considered almost equivalent [9]. Nevertheless, this figure also shows that, for a given mesh size, the dual formulations procedure provides the best PDIV evaluation. The reason of it is still to be found in the presence of numerical errors in the line integral due to (4) not strongly verifying (2a) as illustrate in Fig. 8.In this case, the differences with respect to the measured PDIV are $2.8 \%$ and $9.4 \%$ for the dual formulations procedure and for the line integral from the vector potential computation method respectively. As an illustration, the field lines along which PDs are expected to appear is presented in Fig. 6.

\section{Busbar test piece}

The last application example is a busbar test piece (Fig. 9 and 10). It is composed of two conducting plates of $6 \mathrm{~cm}$ by $11 \mathrm{~cm}$ with a thickness of $1.3 \mathrm{~mm}$. They are insulated from each other by a PET film with a thickness of $190 \mu \mathrm{m}$ and with a relative permittivity of 4.5 . The measured PDIV is equal to $1718 \mathrm{~V}$ (Fig .11).

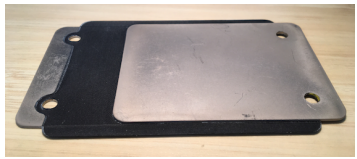

Fig. 9. Busbar test piece

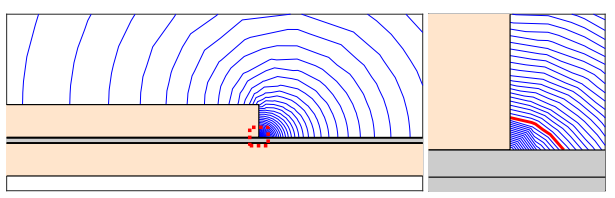

Fig. 10. Field lines distribution in the air surrounding a busbar test piece. (left) zoom on the conductors (right) zoom on the critical field line.

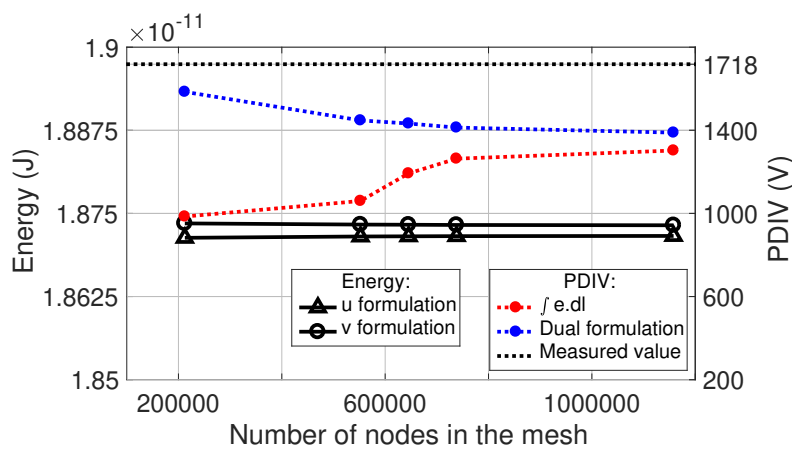

Fig. 11. Energy and PDIV as functions of the number of nodes in the mesh

Several meshes are also used in this case. Fig. 11 show that no matter the mesh, the gap between the two formulations in terms of energy is less than $0.1 \%$. Despite this tiny gap, the same conclusion as the one stated in the previous case can be made. No matter the mesh, the dual formulations procedure always provides the best PDIV determination. Moreover, the PDIV obtained with this procedure is almost the same with the meshes composed of 552000 and 1150000 nodes. Again, the same conclusion on numerical errors can be stated from
Fig. 12. When computations are performed on the mesh composed of 1150000 , the differences with respect to the measured PDIV are $19 \%$ and $24 \%$ for the dual formulations procedure and for the line integral from the vector potential computation method respectively.

Note that this application example is more critical regarding the assumptions mentioned in Section II, especially the one consisting in considering that the field lines are straight. Nevertheless, accounting for this limit and all the uncertainties belonging to the geometry or materials, the dual formulation procedure still provides usable predetermined PDIV.

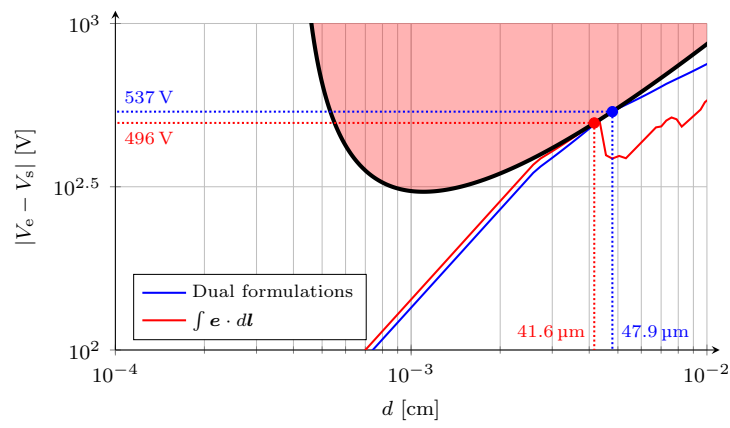

Fig. 12. PDIV determination for a busbar test piece

\section{CONCLUSiON}

In this paper, a numerical method allowing to determine a priori the PDIV in the case of devices with constraining geometries. This method, based on the Paschen's theory and the use of both dual electrostatic formulations, was tested on three application examples. In all three cases, the computed PDIV was in good accordance with the measured one, which makes the proposed method a promising approach for the study of windings or busbars.

\section{REFERENCES}

[1] M. Riera-Guasp, J. A. Antonino-Daviu, and G.-A. Capolino, "Advances in electrical machine, power electronic, and drive condition monitoring and fault detection: state of the art," IEEE Transactions on Industrial Electronics, vol. 62, no. 3, pp. 1746-1759, Mar. 2015.

[2] C. Abadie, T. Billard, and T. Lebey, "Partial discharges in motor fed by inverter: from detection to winding configuration," IEEE Transactions on Industry Applications, 2018.

[3] F. Paschen, "Ueber die zum funkenübergang in luft, wasserstoff und kohlensäure bei verschiedenen drucken erforderliche potentialdifferenz," Annalen der Physik, vol. 273, no. 5, pp. 69-96, 1889.

[4] M. A. Lieberman and A. J. Lichtenberg, Principles of plasma discharges and materials processing, 2nd ed. John Wiley \& Sons, 2005.

[5] N. Hayakawa and H. Okubo, "Partial discharge characteristics of inverter-fed motor coil samples under ac and surge voltage conditions," IEEE Electrical Insulation Magazine, vol. 21, no. 1, pp. 5-10, Jan. 2005.

[6] E. Sili, J.-P. Cambronne, N. Naude, and R. Khazaka, "Polyimide lifetime under partial discharge aging: effects of temperature, pressure and humidity," IEEE Transactions on Dielectrics and Electrical Insulation, vol. 20, no. 2, pp. 435-442, Apr. 2013.

[7] P. Dular, C. Geuzaine, F. Henrotte, and W. Legros, "A general environment for the treatment of discrete problems and its application to the finite element method," IEEE Transactions on Magnetics, vol. 34, no. 5, pp. 3395-3398, 1998.

[8] Z. Ren, "A 3D vector potential formulation using edge element for electrostatic field computation," IEEE Transactions on Magnetics, vol. 31, no. 3, pp. 1520-1523, May 1995.

[9] Z. Ren and H. Qu, "Investigation of the complementarity of dual eddy current formulations on dual meshes," IEEE Transactions on Magnetics, vol. 46, no. 8, pp. 3161-3164, Aug. 2010. 